\title{
160-Gb/s Silicon All-Optical Packet Switch for Buffer-less Optical Burst Switching
}

Hu, Hao; Ji, Hua; Pu, Minhao; Galili, Michael; Yvind, Kresten; Oxenløwe, Leif Katsuo

Published in:

Journal of Lightwave Technology

Link to article, DOI:

10.1109/JLT.2014.2372337

Publication date:

2015

Document Version

Peer reviewed version

Link back to DTU Orbit

Citation (APA):

Hu, H., Ji, H., Pu, M., Galili, M., Yvind, K., \& Oxenløwe, L. K. (2015). 160-Gb/s Silicon All-Optical Packet Switch for Buffer-less Optical Burst Switching. Journal of Lightwave Technology, 33(4), 843-848.

https://doi.org/10.1109/JLT.2014.2372337

\section{General rights}

Copyright and moral rights for the publications made accessible in the public portal are retained by the authors and/or other copyright owners and it is a condition of accessing publications that users recognise and abide by the legal requirements associated with these rights.

- Users may download and print one copy of any publication from the public portal for the purpose of private study or research.

- You may not further distribute the material or use it for any profit-making activity or commercial gain

- You may freely distribute the URL identifying the publication in the public portal

If you believe that this document breaches copyright please contact us providing details, and we will remove access to the work immediately and investigate your claim 


\title{
160-Gb/s Silicon All-Optical Packet Switch for Buffer-less Optical Burst Switching
}

\author{
Hao Hu, Hua Ji, Minhao Pu, Michael Galili, Kresten Yvind, and Leif Katsuo Oxenløwe
}

\begin{abstract}
We experimentally demonstrate a 160-Gb/s Ethernet packet switch using an 8.6-mm-long silicon nanowire for optical burst switching, based on cross phase modulation in silicon. One of the four packets at the bit rate of $160 \mathrm{~Gb} / \mathrm{s}$ is switched by an optical control signal using a silicon based $1 \times 1$ all-optical packet switch. Error free performance (BER $<1 E-9)$ is achieved for the switched packet. The use of optical burst switching protocols could eliminate the need for optical buffering in silicon packet switch based optical burst switching, which might be desirable for highspeed interconnects within a short-reach and small-scale network, such as board-to-board interconnects, chip-to-chip interconnects, and on-chip interconnects.
\end{abstract}

Index Terms-All-optical signal processing, cross phase modulation, optical burst switching (OBS), optical packet switching (OPS), optical time division multiplexing (OTDM), photonic switching, silicon photonics.

\section{$\mathbf{T}$} HE data traffic within Internet data centers and highperformance computing systems have been consistently growing over the past two decades [1]. The very high aggregate bandwidth demands of these systems have opened up opportunities for optics to compete with electronic interconnects, from rack-to-rack interconnects, chip-to-chip interconnects to on-chip interconnects [2]. In order to meet network bandwidth demands, $100 \mathrm{~Gb}$ Ethernet has been adopted by the new IEEE 802.3ba standards [3], however it is quite likely that network traffic will push it even further [4]. Silicon nanophotonics is a promising technology for low-power and costeffective optical interconnects, due to its ultra-compactness, broad working bandwidth, high-speed operation, integration potential with electronics and complementary metal-oxidesemiconductor (CMOS) compatibility allowing cheap mass production [5]-[10]. In addition, silicon based optical signal processing functionalities where many bits are processed in a compact and integrated device without optical-electrical-optical (OEO) conversion has been identified as a potentially energyefficient solution [11].

Current networks use optical circuit switching (OCS) for optical cross-connect, where a lightpath needs to be established

Manuscript received August 15, 2014; revised October 21, 2014; accepted November 15, 2014. This work was supported by the Danish Research Council under the Terabit Ethernet on Silicon Photonic Chips Project, the NESTOR Project and the SiMOF Project, and European Research Council under the SOCRATES Project.

The authors are with the DTU Fotonik, Technical University of Denmark, DK-2800 Kgs. Lyngby, Denmark (e-mail: huhao@fotonik.dtu. dk; huji@fotonik.dtu.dk; mipu@fotonik.dtu.dk; mgal@fotonik.dtu.dk; kryv@ fotonik.dtu.dk; 1kox@ fotonik.dtu.dk).

Color versions of one or more of the figures in this paper are available online at http://ieeexplore.ieee.org.

Digital Object Identifier 10.1109/JLT.2014.2372337 from a source node to a destination node using a physical path. The OCS is suitable for large, stable and long duration traffic flows, where the lightpath setup time is much less than the data duration. However, the internet traffic has been recognized as consisting of a small number of large and long duration traffic flows and many small traffic flows, and exhibits a bursty nature [12]. The OCS is not ideal for bursty traffic, where the data transmission might not have a long duration relative to the setup time of the lightpath. To address the bursty internet traffic, optical packet switching (OPS) and optical burst switching (OBS) have been proposed as excellent candidates for high-speed interconnects, due to their better flexibility, resource utilization, functionality and granularity [13]-[18]. In an OPS network, an optical packet is sent along with its header. While the header is processed by a switching node, the packet needs to be buffered in the optical domain. The main challenge of OPS is lack of a practical optical buffer. In an OBS network, the burst header cell (BHC) is transmitted separately ahead of the transmission of a data burst to control the switching fabric and establish a path for the burst. The BHC contains the usual header information and the burst length. The data burst usually contains multiple packets. OBS can eliminate the need for a data burst to be buffered at the switching node by just waiting for the BHC to be processed. A major challenge of OBS is the data channel reservation protocol. Several protocols have been proposed to schedule bursts efficiently while achieving a high lightpath or bandwidth utilization at the same time, such as tell-and-go (TAG) and just-enough-time [14], [16].

Combination of silicon photonics and optical packet or burst switching might be a desirable technique for high-speed interconnects. Especially, memory devices (such as electronic random access memories (RAM)) are envisioned to be CMOSintegrated in a single silicon photonic chip [7]. Therefore, high speed $(>100 \mathrm{~Gb} / \mathrm{s})$ silicon chips based OPS/OBS are very promising.

Using a silicon nanowire, we have demonstrated a $160 \mathrm{~Gb} / \mathrm{s}$ packet switch, which can be used in OPS [18]. In this paper, we show that a silicon-based $160 \mathrm{~Gb} / \mathrm{s}$ packet switch can also be used for OBS, and optical buffering could be avoided if a telland-wait (TAW) or TAG protocol is applied. In section II, we describe the working principle of the TAW or TAG protocol and compare the silicon based OBS with the $\mathrm{N} \times \mathrm{N}$ silicon based switch matrix. In section III, the design and the characteristics of the silicon nanowire are presented. In section IV, we describe the working principle of cross phase modulation (XPM) in silicon and its application for packet switching. In addition, we show that the silicon-based $1 \times 1$ all-optical packet switch could be upgraded to $1 \times \mathrm{N}$ all-optical packet switch if a fast tuning laser is used. In Sections V and VI, we show the experimental 


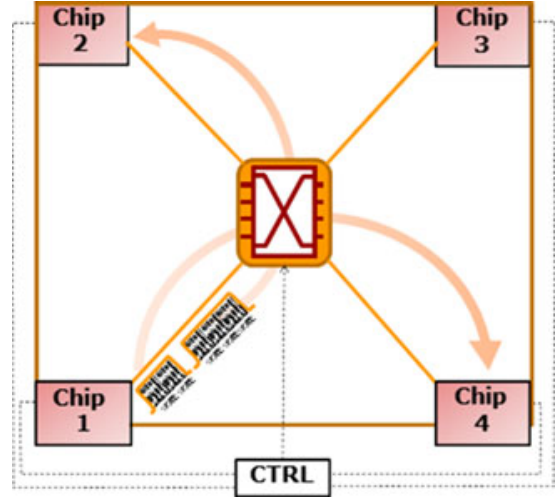

Fig. 1. Short-reach and small-scale network scenario using silicon based OBS.

setup and results of the $160 \mathrm{~Gb} / \mathrm{s}$ all-optical packet switch for OBS. We experimentally demonstrate a $160 \mathrm{~Gb} / \mathrm{s}$ packet switch using an 8.6-mm long silicon nanowire based on XPM. One of four packets at the bit rate of $160 \mathrm{~Gb} / \mathrm{s}$ is switched by an optical control signal. Error free performance (BER $<1 \mathrm{E}-9)$ is achieved for the switched packet.

\section{TAW AND TAG}

In the scenario of short-reach and small-scale interconnects (as shown in Fig. 1), such as interconnections among servers, boards and even chips, optical packets could be stored in the electronic domain using electronic RAM and will not be converted into the optical domain and transmitted until the switching node is ready for the packet. In this case, a two-way reservation protocol such as TAW can be applied [19], [20]. Since the end nodes are close to each other, both BHC and confirmation from the switching node can be sent in the electronic domain with negligible latency and avoiding OEO conversion, which is different in the case of conventional OBS. Another different feature is that the data payload of a burst could be either a short packet or several accumulated long packets, which makes the switching more flexible but requires faster switching speed. In comparison with conventional OPS, which can be used in the large-scale interconnects but requires label processor and packet buffer [21]-[23], our proposed scheme addresses the scenario of short-reach interconnects, and no label is transmitted together with the packet and packet buffer can be avoided.

As shown in Fig. 1, when an ingress end node (EN, e.g., Chip 1) needs to transfer a packet, it first sends a setup message (i.e., BHC) to the control plane. When the control plane receives the setup message, a virtual path will be established towards its egress EN (e.g., Chip 2 or Chip 4) during the data payload transmission if the switching node is free, and then the control plane will send a confirmation message to the ingress EN. Once the ingress EN receives the confirmation message, the data payload stored at the electronic RAM of the ingress EN will be immediately converted into an optical burst and then sent to the egress EN. The virtual path will be automatically released according to the BHC. If more than one ingress EN need to transfer data payload at the same time or the switching node is busy, the data payload at the ingress ENs is still stored

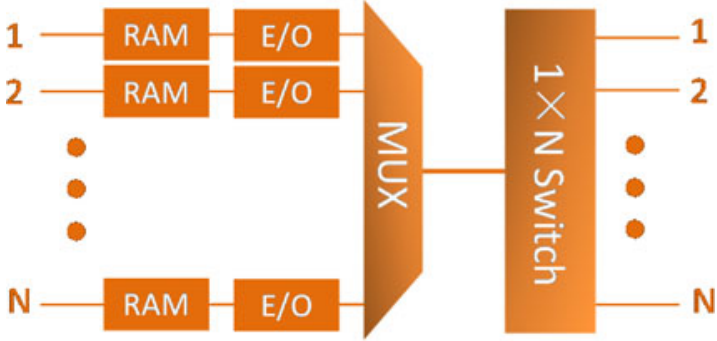

(a)

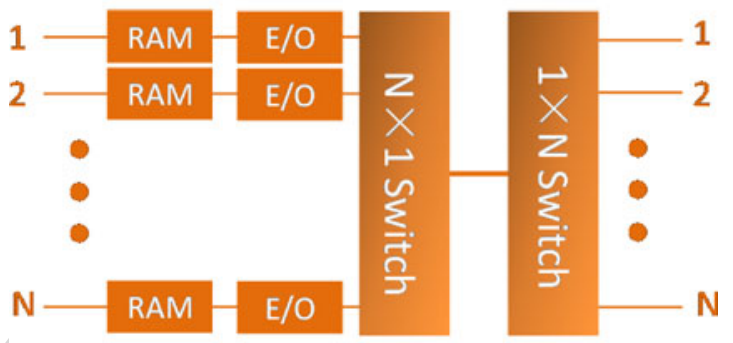

(b)

Fig. 2. Schematic architecture of silicon packet switch based OBS using (a) TAW and (b) TAG.

in the electronic RAM and a waiting list will be established in the control plane. The data payload will wait for the transfer according to the sequence of the list. The sequence of the list depends on the priority based class-of-service. Even if the packet blocking probability rises with higher data load, higher class services experience relatively lower blocking probability compared to lower class services [15]. Fig. 2(a) shows a possible architecture of a silicon packet switch based optical burst switch using TAW protocol. Only one of $\mathrm{N}$ ingress ENs will receive the confirmation message at a time and send data payload to the $1 \times \mathrm{N}$ switch through a multiplexer, which could be a coupler. The $1 \times \mathrm{N}$ switch will switch the data payload to its egress EN according to the BHC.

Another scheme is TAG, which is a one-way reservation protocol and requires no acknowledgement from the switching node before sending the data payload. When an ingress EN has a data payload to transfer, it sequentially sends a setup message to the control plane and an optical burst to the optical switch with a guard time in between. The guard time is at least equal to or more than the time interval needed for setup of a virtual path inside the switching node. This allows the optical switch to be set before the packet arrives. If the switching node is free when it receives the setup message, a virtual path will be set up for the packet transfer and a successful message will be sent back to the ingress EN. If the switching node is busy when it receives the setup message, the optical packet sent from the ingress EN will be discarded and a fail message will be sent back to the ingress EN. If the ingress EN receives the successful message, the electronic RAM storing the data payload will be released. If the ingress EN receives the fail message, it will send the setup message and the optical burst again. Fig. 2(b) shows a possible architecture of silicon packet switch based OBS using TAG protocol. If the control plane receives a setup message from an ingress EN and the switching node is free at the time, the first 

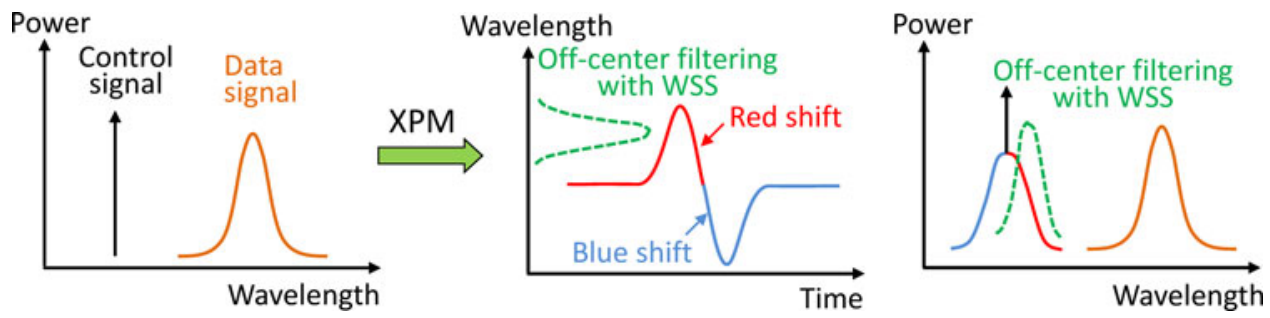

Fig. 3. Operation principle of the XPM in a silicon nanowire with subsequent off-center filtering.

$\mathrm{N} \times 1$ switch will connect to the ingress $\mathrm{EN}$ and allow the data payload to enter, and the second $1 \times \mathrm{N}$ switch will switch to its egress EN according to the BHC. If the switching node is busy when the control plane receives the setup message, the first $1 \times \mathrm{N}$ switch will not connect to the ingress $\mathrm{EN}$ and the data payload sent from the ingress EN will be discarded.

Compared to an $\mathrm{N} \times \mathrm{N}\left(N \in 2^{\mathrm{n}}, n=1,2,3, \ldots\right)$ silicon based switch matrix with a granularity of the optical paths, silicon based OBS with a granularity of optical packets is more flexible and could have smaller footprint and less power consumption. For the $\mathrm{N} \times \mathrm{N}$ switch matrix based on path-independent insertion loss and "switch-and-select" topology, the total number of Mach-Zehnder interferometer (MZI) switches is $2 \times \mathrm{N} \times \mathrm{N}$ and $2 \times \mathrm{N} \times(\mathrm{N}-1)$, respectively [24], [25]. Assuming MZI switches are also used in the $1 \times \mathrm{N}$ switch, as shown in Fig. 2, the total number of MZI switches for the TAW and TAG are $\mathrm{N}-1$ and $2 \times(\mathrm{N}-1)$, respectively. In addition, no intersections are needed for the $1 \times \mathrm{N}$ switch, and therefore there will be no crosstalk.

A main requirement for the silicon packet switch is that the switching speed of the optical switches should be fast enough in order to introduce less latency and lower blocking probability, and therefore the switching time should preferably be no more than a few nanoseconds.

\section{SILICON NANOWIRE}

The key device for the silicon all-optical packet switch is a dispersion engineered 8.6- $\mathrm{mm}$ long silicon straight waveguide, which includes tapering sections for low loss interfacing with optical fiber [26]. The main waveguide section is $\sim 8 \mathrm{~mm}$ long and has a cross-sectional dimension of $240 \mathrm{~nm} \times 450 \mathrm{~nm}$ while the tapering sections are $\sim 0.3 \mathrm{~mm}$ long each. The width at the end of the silicon nanowire is tapered from $450 \mathrm{~nm}$ to a tiny tip end of $40 \mathrm{~nm}$ so that the guided mode will expand into a polymer waveguide, surrounding the silicon-on-insulator (SOI) waveguide and the taper. The device has an SOI structure, with the silicon waveguide placed on a $\mathrm{SiO}_{2} / \mathrm{Si}$ substrate. The measured propagation loss is $4.3 \mathrm{~dB} / \mathrm{cm}$ and the fiber-to-fiber loss of the device is $6.8 \mathrm{~dB}$.

\section{XPM In A Silicon NanOwiRe FOR Packet SWITCH}

XPM is an ultrafast optical Kerr effect, with a response time of a few fs. Fig. 3 shows the operation principle of XPM in a silicon nanowire with subsequent off-center filtering. The pump pulse can modulate the refractive index of the silicon waveguide, which results in phase modulation on the co-propagating con- tinuous wave $(\mathrm{CW})$ probe [27]. The phase modulation will then result in transient chirp on the $\mathrm{CW}$ probe. The leading edges of the pump pulse will generate red-shift chirp, whereas the trailing edge of the pump pulse will generate blue-shift chirp. The blue shifted and red shifted sidebands are generated as a result of the chirp. If an off-center filter is used to extract either the blue shifted sideband or the red shifted sideband, the XPM-induced phase modulation can be converted into amplitude modulation. When an RZ-OOK data signal is used as the pump, the generated sideband can pass through the off-center filter in the presence of a " 1 " bit of the pump, whereas no generated sideband results in no transmission through the off-center filter in the presence of a " 0 " bit of the pump. Using a CW probe, the XPM in silicon with subsequent off-center filtering has been used for forward error correction supported $150 \mathrm{~Gb} / \mathrm{s}$ wavelength conversion, $10 \mathrm{~Gb} / \mathrm{s}$ tuneable wavelength conversion, $40 \mathrm{~Gb} / \mathrm{s}$ regenerative wavelength conversion and $160 \mathrm{~Gb} / \mathrm{s}$ all-optical data modulator [27]-[30]. If the probe is gated in time (with a gating time slightly larger than the packet duration), XPM in silicon with subsequent off-center filtering, can be used for packet switching with ultrafast response time. Fig. 4 shows an illustration of packet switch based on the XPM in silicon with subsequent off-center filtering using gated probe light. For the $1 \times 1$ packet switch, 1 out of 4 data packets $\left(\lambda_{D}\right)$ is switched out at the wavelength of $\lambda_{\mathrm{C}}+\Delta \lambda$ when the control signal $\left(\lambda_{\mathrm{C}}\right)$ is set to be on. When the control signal is off, no light is generated at the wavelength of $\lambda_{\mathrm{C}}+\Delta \lambda$. For the $1 \times \mathrm{N}$ packet switch, a wavelength selective switch (WSS) with different wavelengths at different outputs should be used. When the wavelength of the control signal is fast tuned, the incoming packet could be switched to different outputs of the WSS. The tuning speed of the tunable laser depends on the guard band between packets, which is typically on the order of several nanoseconds to several microseconds. This requirement of tuning speed could be relaxed if the granularity of the packet becomes large, i.e., introducing large enough guard band $(\sim \mu$ s) by switching a long packet or several aggregated short packets. If the first packet of four data packets needs to be switched to port 1 of the WSS, the wavelength of the control signal should be tuned to be $\lambda_{\mathrm{C} 1}$; if the second packet of four data packets needs to be switched to port 4 of the WSS, the wavelength of the control signal should be tuned to be $\lambda_{\mathrm{C} 4}$. Compared to the $1 \times \mathrm{N}$ packet switch based on cascaded MZI switches, which needs $\mathrm{N}-1$ active switch, the XPM based $1 \times \mathrm{N}$ packet switch only needs an active fast tunable laser followed by passive filtering. Based on the $1 \times \mathrm{N}$ packet switch, the TAW and TAG protocol can be realized, as shown in Fig. 2. 


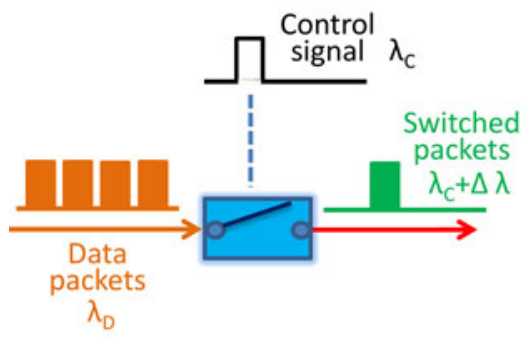

(a)

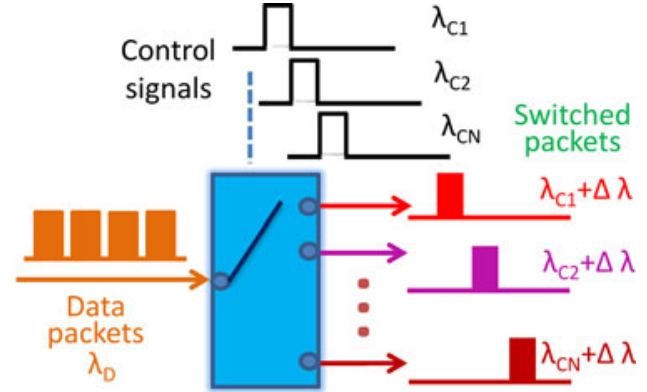

(b)

Fig. 4. Illustration of packet switch. (a) One out of four data packets is switched out using $1 \times 1$ packet switch when the control signal is set to be on; (b) One of the input data packets is switched to different path using $1 \times \mathrm{N}$ packet switch when the wavelength of the control signal is tuned. Blue box: WSS with different wavelengths at different outputs.

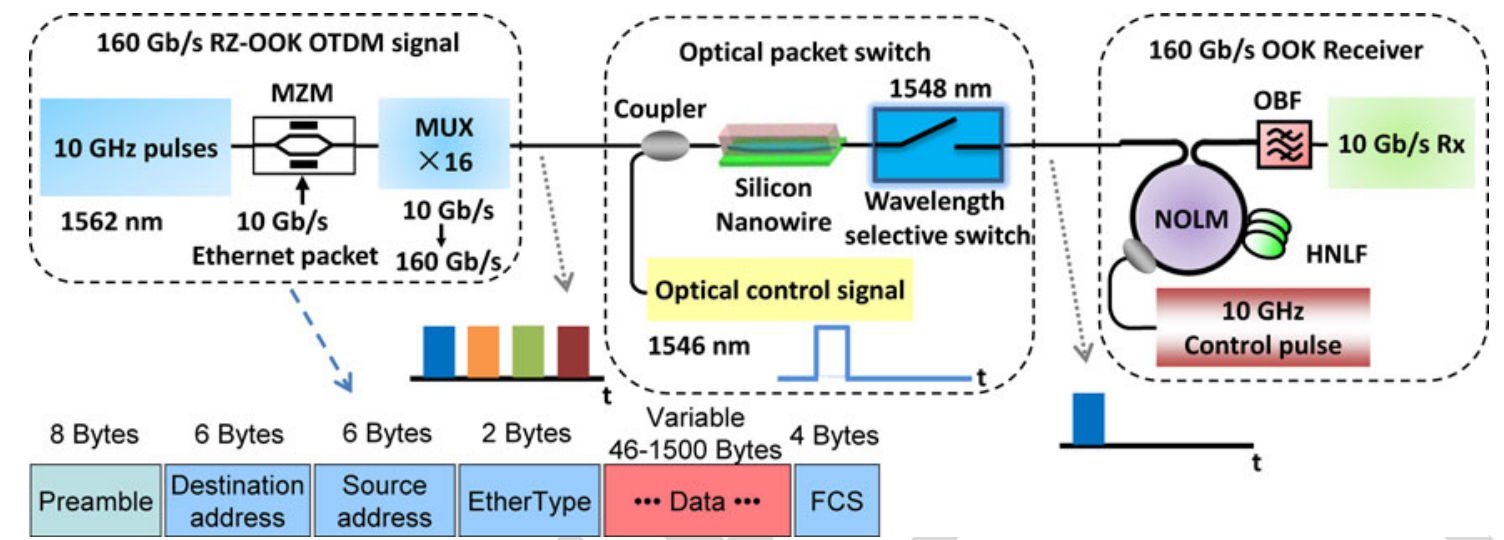

Fig. 5. Experimental setup for the $160 \mathrm{~Gb} / \mathrm{s}$ all-optical packet switch using a silicon nanowire followed by a WSS.

\section{EXPERIMENTAL SETUP}

The experimental setup for the $160 \mathrm{~Gb} / \mathrm{s}$ silicon packet switch is shown in Fig. 5. It mainly includes a $160 \mathrm{~Gb} / \mathrm{s}$ RZ-OOK transmitter, a $1 \times 1$ silicon based packet switch and a $160 \mathrm{~Gb} / \mathrm{s}$ on-off keying (OOK) receiver. The erbium-glass oscillating pulse-generating laser produces $10 \mathrm{GHz}$ pulses at $1542 \mathrm{~nm}$ with a 1.5-ps full-width at half-maximum pulse width. The spectrum of the pulses is broadened in a 400-m dispersion-flattened highly nonlinear fibre (DF-HNLF, dispersion coefficient $\mathrm{D}=$ $-0.45 \mathrm{ps} / \mathrm{nm} / \mathrm{km}$ and dispersion slope $\mathrm{S}=0.006 \mathrm{ps} / \mathrm{nm}^{2} / \mathrm{km}$ at $1550 \mathrm{~nm}$, nonlinear coefficient $\gamma=10.5 \mathrm{~W}^{-1} \mathrm{~km}^{-1}$ ) due to self-phase modulation [31], [32]. The broadened spectrum is filtered at $1562 \mathrm{~nm}$ with a 5-nm optical bandpass filter (OBF) to generate the $10 \mathrm{GHz}$ pulses for the data signal and is also filtered at $1538 \mathrm{~nm}$ using a $1 \mathrm{~nm}$ OBF to obtain the $10 \mathrm{GHz}$ control pulses used in the OTDM demultiplexing. The generated $10 \mathrm{GHz}$ pulses at $1562 \mathrm{~nm}$ are OOK modulated in a MachZehnder modulator (MZM) using a software defined pattern to generate $10 \mathrm{~Gb} / \mathrm{s}$ Ethernet packets. As shown in Fig. 5, the Ethernet packet with a maximum standardized size of 1518 bytes consists of a preamble, a destination address, a source address, an Ethertype, payload data and a frame check sequence [33]. The packets have duration of $2.19 \mu \mathrm{s}$, consisting of $1.22 \mu \mathrm{s}$ of data payload separated by a $0.97 \mu$ s guard band. The generated 10 $\mathrm{Gb} / \mathrm{s}$ Ethernet packet is multiplexed in time using a passive fiberdelay multiplexer $(\mathrm{MUX} \times 16$ ) to generate the $160 \mathrm{~Gb} / \mathrm{s}$ signal.
In the $(1 \times 1)$ silicon based optical packet switch, the gen- 283 erated $160 \mathrm{~Gb} / \mathrm{s}$ optical packet is amplified by an EDFA, then 284 filtered by a $5 \mathrm{~nm} \mathrm{OBF}$ and finally launched into the silicon 285 nanowire through a 3-dB optical coupler. The optical control 286 signal is generated from a CW light at $1546 \mathrm{~nm}$, which is mod- 287 ulated by an electrical control signal in a MZM. The electrical 288 control signal is generated according to the BHC of the burst, 289 which has duration of $1.5 \mu \mathrm{s}$ and repetition rate of $\sim 114 \mathrm{kHz} 290$ in order to switch one of the four packets. The optical control 291 signal is also launched into the silicon nanowire through the 292 second input of the 3-dB coupler. Fig. 6 shows the waveforms 293 of the packets and the control signal. The launched average 294 power for the data and the control are 13.5 and $8.5 \mathrm{dBm}$, respec- 295 tively, which corresponds to the energy consumption of 140 and 296 $44 \mathrm{fJ} / \mathrm{bit}$. Since the total launched power is well below the two 297 photon absorption (TPA) threshold [6], the TPA and resulting 298 carrier effects are negligible. The polarizations of the data signal 299 and control signal are both aligned to transverse-electric polar- 300 izations into the silicon waveguide. The optical packets modu- 301 late the refractive index of the silicon waveguide and generate 302 XPM on the control signal. Only if the control signal presents 303 "1" level and aligned in time with a packet, the control signal will 304 be phase modulated by the optical packet and be converted into 305 an amplitude modulated signal by passing through an off-center 306 filter. Note that the refractive index of the silicon waveguide 307 could also be modulated by an electrical signal [8], therefore, 308 


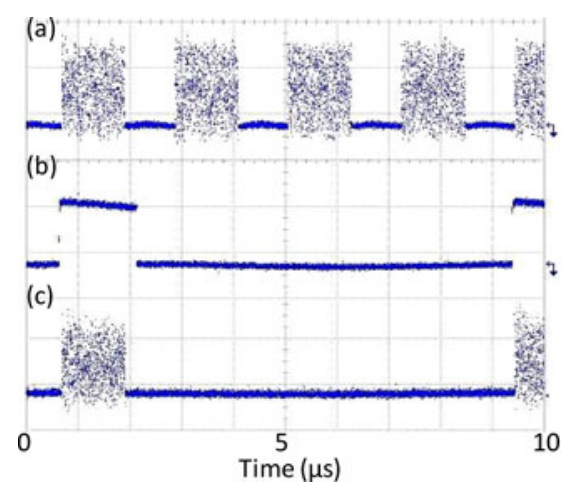

Fig. 6. Oscilloscope traces (a) $160 \mathrm{~Gb} / \mathrm{s}$ optical packets at the input of the silicon nanowire; (b) optical control signal; (c) one out of four packets switched at the output of the silicon nanowire and WSS.

in principle the scheme could also work if the electrical signal is directly employed on the silicon chip. At the output of the silicon nanowire, a WSS with the center wavelength of $1548 \mathrm{~nm}$ is used to filter out the red-shifted sideband of the control signal and select the switched packet.

The 4-to-1 switched $160 \mathrm{~Gb} / \mathrm{s}$ packet was detected using a $160 \mathrm{~Gb} / \mathrm{s}$ OOK receiver, which consists of a nonlinear optical loop mirror (NOLM) based OTDM demultiplexer, a 0.9-nm filter, a photo detector (PD) and an error analyzer. The NOLM is used to OTDM demultiplex the $160 \mathrm{~Gb} / \mathrm{s}$ packet down to $10 \mathrm{~Gb} / \mathrm{s}$ packets based on the XPM in a $50 \mathrm{~m}$ long HNLF. Finally, the demultiplexed $10 \mathrm{~Gb} / \mathrm{s}$ optical packet was detected using a PD and the performance was evaluated using an error analyzer.

\section{EXPERIMENTAL RESULTS}

Fig. 6 shows the dynamic operation of $160 \mathrm{~Gb} / \mathrm{s}$ silicon packet switch. When the control plane receives BHC information, a control signal was generated to drive the silicon based all-optical switch. In this case, one of four packets needs to be switched; therefore, the targeted packet was aligned in time with the optical control signal, as shown in Fig. 6(a) and (b). At the output of the packet switch, the targeted packet was successfully switched with an extinction ratio of $\sim 18 \mathrm{~dB}$, as shown in Fig. 6(c). Fig. 7 shows optical sampling oscilloscope (OSO) eye diagrams of $160 \mathrm{~Gb} / \mathrm{s}$ original packets and 1-of-4 switched packet. The switched packet has an open and clear eye diagram after the packet switch. Actually, some " 0 " level noise on the original packets was also removed after the $1 \times 1$ packet switch, resulting from the regenerative characteristics of the off-center filtering [29].

The spectra at the input and the output of the silicon nanowire are shown in Fig. 8. XPM on the control signal can be clearly seen (modulation peaks around the control signal) at the output of the silicon nanowire. The blue and red shifted sidebands on the optical control signal were generated from the leading edge and trailing edge of the $160 \mathrm{~Gb} / \mathrm{s}$ optical packets with the modulation format of RZ-OOK, respectively. A WSS centered at $1548 \mathrm{~nm}$ is used to filter out the red-shifted probe light to

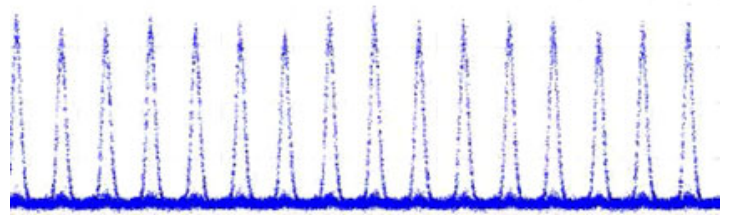

(a)

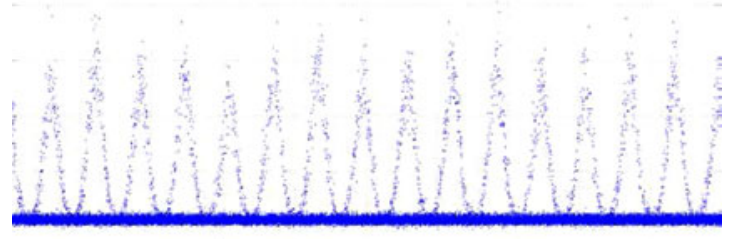

(b)

Fig. 7. (a) OSO eye diagrams of $160 \mathrm{~Gb} / \mathrm{s}$ original packets (b) and 1-of-4 switched packet.

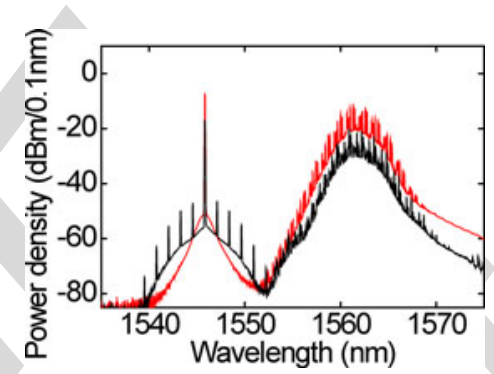

Fig. 8. Optical spectra at the input of the silicon chip (red) and output of the silicon nanowire (black).

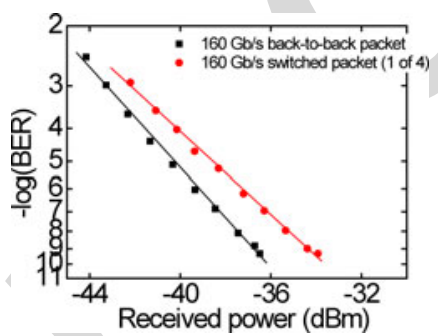

Fig. 9. BER measurements after demultiplexing to $10 \mathrm{~Gb} / \mathrm{s}$ for the $160 \mathrm{~Gb} / \mathrm{s}$ back-to-back packet and for the $160 \mathrm{~Gb} / \mathrm{s}$ 4-to-1 switched packet.

obtain an amplitude modulated $160 \mathrm{~Gb} / \mathrm{s}$ signal and separate the switched packet at $1548 \mathrm{~nm}$ from the original packet at $1562 \mathrm{~nm}$.

The performance of the silicon based $1 \times 1$ all-optical packet switch for the $160 \mathrm{~Gb} / \mathrm{s}$ Ethernet packet was evaluated using BER measurements, as shown in Fig. 9. BER curves are plotted for the $160 \mathrm{~Gb} / \mathrm{s}$ back-to-back packets and for the $160 \mathrm{~Gb} / \mathrm{s}$ 4-to- 1 switched packets. The 4-to- 1 switched $160 \mathrm{~Gb} / \mathrm{s}$ packets achieve an error-free performance (BER $<10^{-9}$ ) with a power penalty of $\sim 2.5 \mathrm{~dB}$ compared to the back-to-back case. The measured penalty is partly attributed to the pulse broadening due to the filtering effect induced by the WSS, and partly attributed to the OSNR degradation after the packet switch due to the limited phase modulation on the control signal. 


\section{DISCUSSION AND CONCLUSION}

We have successfully demonstrated a $160 \mathrm{~Gb} / \mathrm{s}$ silicon based $1 \times 1$ all-optical packet switch, based on XPM in a silicon nanowire. This scheme could be upgraded to a $1 \times \mathrm{N}$ all-optical packet switch if a fast tunable laser is used. The silicon packet switch could be used either for OPS or OBS without optical buffering if the TAW or TAG protocol is used. The 4-to-1 switched $160 \mathrm{~Gb} / \mathrm{s}$ Ethernet packet shows error free performance (BER $<1 \mathrm{E}-9$ ), and holds great promise for future photonic switching of ultra-fast data signals.

\section{REFERENCES}

[1] Y.A. Vlasov, "Silicon CMOS-integrated nano-photonics for computer and data communications beyond 100G," IEEE Commun. Mag., vol. 50, no. 2, pp. s67-s72, Feb. 2012.

[2] P. W. Coteus, J. U. Knickerbocker, C. H. Lam, and Y. A. Vlasov, "Technologies for exascale systems," IBM J. Res. Develop., vol. 55, no. 5, pp. 14:1-14:12, Sep./Oct. 2011. EEE P802.3ba. [Online]. Available: http://www.ieee802.org/3/

PEE 802.3 industry connections ethernet bandwidth assessment Ad Hoc. Dnline]. Available: http://www.ieee802.org/3/ad_hoc/bwa/index.html.

[5] M. A. Foster, A. C. Turner, J. E. Sharping, B. S. Schmidt, M. Lipson, and A. L. Gaeta, "Broad-band optical parametric gain on a silicon photonic chip," Nature, vol. 441, pp. 960-963, 2006.

[6] H. Hu, H. Ji, M. Galili, M. Pu, H. C. H. Mulvad, L. K. Oxenløwe, K. Yvind, J. M. Hvam, and P. Jeppesen, "Ultra-high-speed wavelength conversion in a silicon photonic chip," Opt. Exp., vol. 19, no. 21, pp. 19886-19894, 2011.

[7] C. Batten, A. Joshi, J. Orcutt, A. Khilo, B. Moss, C. W. Holzwarth, M. A. Popovic, H. Li, H. Smith, J. L. Hoyt, F. X. Kartner, R. J. Ram, V. Stojanovic, and K. Asanovic, "Building many-core processor-toDRAM networks with monolithic CMOS silicon photonics," IEEE Micro, vol. 29, no. 4, pp. 8-21, Jul./Aug. 2009.

[8] P. Dong, X. Liu, C. Sethumadhavan, L. L. Buhl, R. Aroca, Y. Baeyens, and Y. Chen, "224-Gb/s PDM-16-QAM modulator and receiver based on silicon photonic integrated circuits," presented at the Opt. Fiber Commun. Conf., Nat. Fiber Opt. Eng., Anaheim, CA, USA, 2013, Paper PDP5C.6.

[9] H. Ji, M. Pu, H. Hu, M. Galili, L. K. Oxenløwe, K. Yvind, J. M. Hvam, and P. Jeppsen, "Optical waveform sampling and error-free demultiplexing of $1.28 \mathrm{~Tb} / \mathrm{s}$ serial data in a nanoengineered silicon waveguide," J. Lightw. Technol., vol. 29, no. 4, pp. 426-431, Feb. 2011.

[10] H. C. Hansen Mulvad, E. Palushani, H. Hu, H. Ji, M. Lillieholm, M. Galili, A. T. Clausen, M. Pu, K. Yvind, J. M. Hvam, P. Jeppesen, and L. K. Oxenløwe, "Ultra-high-speed optical serial-to-parallel data conversion by time-domain optical Fourier transformation in a silicon nanowire," Opt. Exp., vol. 19, pp. B825-B835, 2011.

[11] J. Leuthold, C. Koos, and W. Freude, "Nonlinear silicon photonics," Nature Photon., vol. 4, pp. 535-544, 2010.

[12] W. Stallings, High-Speed Networks. Englewood Cliffs, NJ, USA: PrenticeHall, 1998.

[13] S. Yao, S. J. B. Yoo, B. Mukherjee, and S. Dixit, "All-optical packet switching for metropolitan area networks: Opportunities and challenges," IEEE Commun. Mag., vol. 39, no. 3, pp. 142-148, Mar. 2001.

[14] C. M. Qiao and M. S. Yoo, "Optical burst switching (OBS)—A new paradigm for an optical Internet," J. High Speed Netw., vol. 8, no. 1, pp. 69-84, 1999.

[15] M. Yoo, C. M. Qiao, and S. Dixit, "Optical burst switching for service differentiation in the next-generation optical internet," IEEE Commun. Mag., vol. 39, no. 2, pp. 98-104, Feb. 2001.

[16] S. J. B. Yoo, "Optical packet and burst switching technologies for the future photonic internet," J. Lightw. Technol., vol. 24, no. 12, pp. 4468 -4492 , Dec. 2006.

[17] F. Gomez-Agis, H. Hu, J. Luo, H. C. H. Mulvad, M. Galili, N. Calabretta, L. K. Oxenløwe, H. J. S. Dorren, and P. Jeppesen, "Optical switching and detection of $640 \mathrm{Gbits} / \mathrm{s}$ optical time-division multiplexed data packets transmitted over $50 \mathrm{~km}$ of fiber," Opt. Lett., vol. 36, pp. 3473-3475, 2011.
[18] H. Hu, H. Ji, M. Galili, M. Pu, K. Yvind, P. Jeppesen, and L. K. Oxenløwe, "160 Gbit/s optical packet switching using a silicon chip," in Proc. IEEE Photon. Conf., Sep. 2012, pp. 915-916.

[19] P. E. Boyer and D. P. Tranchier, "A reservation principle with applications to the ATM traffic control," Comput. Netw. ISDN Syst., vol. 24, pp. 321-334, 1992.

[20] A. Detti and M. Listanti, "Application of tell \& Go and tell \& wait reservation strategies in a optical burst switching network: A performance comparison," in Proc. IEEE Int. Conf. Telecommun., 2001, vol. 2, pp. $540-548$.

[21] N. Calabretta, R. P. Centelles, S. Di Lucente, and H. J. S. Dorren, "On the performance of a large-scale optical packet switch under realistic data center traffic," J. Opt. Commun. Netw., vol. 5, no. 6, pp. 565-573, Jun. 2013.

[22] S. Di Lucente, N. Calabretta, J. A. C. Resing, and H. J. S. Dorren, "Scaling low-latency optical packet switches to a thousand ports," J. Opt. Commun. Netw., vol. 4, no. 9, pp. A17-A28, Sep. 2012.

[23] H. J. S. Dorren, S. Di Lucente, J. Luo, O. Raz, and N. Calabretta, "Scaling photonic packet switches to a large number of ports [invited]," J. Opt. Commun. Netw., vol. 4, no. 9, pp. A82-A89, Sep. 2012.

[24] L. Chen and Y. K. Chen, "Compact, low-loss and low-power $8 \times 8$ broadband silicon optical switch," Opt. Exp., vol. 20, no. 17, pp. 18977-18985, 2012.

[25] K. Suzuki, K. Tanizawa, T. Matsukawa, G. Cong, S. H. Kim, S. Suda, M. Ohno, T. Chiba, H. Tadokoro, M. Yanagihara, Y. Igarashi, M. Masahara, and H. Kawashima, "Ultra-compact Si-wire $8 \times 8$ strictly-non-blocking PILOSS switch," presented at the Eur. Conf. Exhib. Opt. Commun., London, U.K., 2013, Paper PD2.D.2.

[26] M. Pu, L. Liu, H. Ou, K. Yvind, and J. M. Hvam, "Ultra-low-loss inverted taper coupler for silicon-on-insulator ridge waveguide," Opt. Coтmun., vol. 283, pp. 3678-3682, Oct. 2010.

[27] H. Hu, J. Dahl Andersen, A. Rasmussen, B. M. Sørensen, K. Dalgaard, M. Galili, M. Pu, K. Yvind, K. J. Larsen, S. Forchhammer, and L. K. Oxenløwe, "Forward error correction supported $150 \mathrm{Gbit} / \mathrm{s}$ error-free wavelength conversion based on cross phase modulation in silicon," Opt. Exp., vol. 21, pp. 3152-3160, 2013.

[28] W. Astar, J. B. Driscoll, X. Liu, J. I. Dadap, W. M. J. Green, Y. Vlasov, G. M. Carter, and R. M. Osgood, "Tunable wavelength conversion by XPM in a silicon nanowire, and the potential for XPM-multicasting," $J$. Lightw. Technol., vol. 28, no. 17, pp. 2499-2511, Sep. 2010.

[29] A. S. Jensen, H. Hu, H. Ji, M. Pu, L. H. Frandsen, and L. K. Oxenløwe, "All-optical $40 \mathrm{Gbit} / \mathrm{s}$ regenerative wavelength conversion based on crossphase modulation in a silicon nanowire," presented at the IEEE 18th OptoElectron. Commun. Conf. Held Jointly Int. Photon. Switching, Kyoto, Japan, 2013, Paper ThM1-2.

[30] H. Hu, M. Pu, H. Ji, M. Galili, H. C. H. Mulvad, K. Yvind, J. M. Hvam, P. Jeppesen, and L. Oxenlowe, " $160 \mathrm{~Gb} / \mathrm{s}$ silicon all-optical data modulator based on cross phase modulation," presented at the Optical Fiber Commun. Conf. Expo. Nat. Fiber Opt. Eng. Conf., Los Angeles, CA, USA, 2012, Paper OM2E.2.

[31] H. Hu, H. C. H. Mulvad, M. Galili, E. Palushani, J. Xu, A. T. Clausen, L. K. Oxenløwe, and P. Jeppesen, "Polarization-insensitive $640 \mathrm{~Gb} / \mathrm{s} \mathrm{de}-$ multiplexing based on four wave mixing in a polarization-maintaining fibre loop," J. Lightw. Technol., vol. 28, no. 12, pp. 1789-1795, Jun. 2010.

[32] H. Hu, E. Palushani, M. Galili, H. C. H. Mulvad, A. Clausen, L. K. Oxenløwe, and P. Jeppesen, "640 Gbit/s and $1.28 \mathrm{Tbit} / \mathrm{s}$ polarisation insensitive all optical wavelength conversion," Opt. Exp., vol. 18, pp. 99619966, 2010

[33] H. Hu, J. L. Areal, H. C. H. Mulvad, M. Galili, K. Dalgaard, E. Palushani, A. Clausen, M. S. Berger, P. Jeppesen, and L. K. Oxenløwe, "Synchronization, retiming and time-division multiplexing of an asynchronous 10 Gigabit NRZ Ethernet packet to terabit Ethernet," Opt. Exp., vol. 19, pp. B931-B937, 2011. 


\title{
160-Gb/s Silicon All-Optical Packet Switch for Buffer-less Optical Burst Switching
}

\author{
Hao Hu, Hua Ji, Minhao Pu, Michael Galili, Kresten Yvind, and Leif Katsuo Oxenløwe
}

\begin{abstract}
We experimentally demonstrate a 160-Gb/s Ethernet packet switch using an 8.6-mm-long silicon nanowire for optical burst switching, based on cross phase modulation in silicon. One of the four packets at the bit rate of $160 \mathrm{~Gb} / \mathrm{s}$ is switched by an optical control signal using a silicon based $1 \times 1$ all-optical packet switch. Error free performance (BER $<1 E-9)$ is achieved for the switched packet. The use of optical burst switching protocols could eliminate the need for optical buffering in silicon packet switch based optical burst switching, which might be desirable for highspeed interconnects within a short-reach and small-scale network, such as board-to-board interconnects, chip-to-chip interconnects, and on-chip interconnects.
\end{abstract}

Index Terms-All-optical signal processing, cross phase modulation, optical burst switching (OBS), optical packet switching (OPS), optical time division multiplexing (OTDM), photonic switching, silicon photonics.

\section{$\mathbf{T}$} HE data traffic within Internet data centers and highperformance computing systems have been consistently growing over the past two decades [1]. The very high aggregate bandwidth demands of these systems have opened up opportunities for optics to compete with electronic interconnects, from rack-to-rack interconnects, chip-to-chip interconnects to on-chip interconnects [2]. In order to meet network bandwidth demands, $100 \mathrm{~Gb}$ Ethernet has been adopted by the new IEEE 802.3ba standards [3], however it is quite likely that network traffic will push it even further [4]. Silicon nanophotonics is a promising technology for low-power and costeffective optical interconnects, due to its ultra-compactness, broad working bandwidth, high-speed operation, integration potential with electronics and complementary metal-oxidesemiconductor (CMOS) compatibility allowing cheap mass production [5]-[10]. In addition, silicon based optical signal processing functionalities where many bits are processed in a compact and integrated device without optical-electrical-optical (OEO) conversion has been identified as a potentially energyefficient solution [11].

Current networks use optical circuit switching (OCS) for optical cross-connect, where a lightpath needs to be established

Manuscript received August 15, 2014; revised October 21, 2014; accepted November 15, 2014. This work was supported by the Danish Research Council under the Terabit Ethernet on Silicon Photonic Chips Project, the NESTOR Project and the SiMOF Project, and European Research Council under the SOCRATES Project.

The authors are with the DTU Fotonik, Technical University of Denmark, DK-2800 Kgs. Lyngby, Denmark (e-mail: huhao@fotonik.dtu. dk; huji@fotonik.dtu.dk; mipu@fotonik.dtu.dk; mgal@fotonik.dtu.dk; kryv@ fotonik.dtu.dk; lkox@ fotonik.dtu.dk).

Color versions of one or more of the figures in this paper are available online at http://ieeexplore.ieee.org.

Digital Object Identifier 10.1109/JLT.2014.2372337 from a source node to a destination node using a physical path. The OCS is suitable for large, stable and long duration traffic flows, where the lightpath setup time is much less than the data duration. However, the internet traffic has been recognized as consisting of a small number of large and long duration traffic flows and many small traffic flows, and exhibits a bursty nature [12]. The OCS is not ideal for bursty traffic, where the data transmission might not have a long duration relative to the setup time of the lightpath. To address the bursty internet traffic, optical packet switching (OPS) and optical burst switching (OBS) have been proposed as excellent candidates for high-speed interconnects, due to their better flexibility, resource utilization, functionality and granularity [13]-[18]. In an OPS network, an optical packet is sent along with its header. While the header is processed by a switching node, the packet needs to be buffered in the optical domain. The main challenge of OPS is lack of a practical optical buffer. In an OBS network, the burst header cell (BHC) is transmitted separately ahead of the transmission of a data burst to control the switching fabric and establish a path for the burst. The BHC contains the usual header information and the burst length. The data burst usually contains multiple packets. OBS can eliminate the need for a data burst to be buffered at the switching node by just waiting for the BHC to be processed. A major challenge of OBS is the data channel reservation protocol. Several protocols have been proposed to schedule bursts efficiently while achieving a high lightpath or bandwidth utilization at the same time, such as tell-and-go (TAG) and just-enough-time [14], [16].

Combination of silicon photonics and optical packet or burst switching might be a desirable technique for high-speed interconnects. Especially, memory devices (such as electronic random access memories (RAM)) are envisioned to be CMOSintegrated in a single silicon photonic chip [7]. Therefore, high speed $(>100 \mathrm{~Gb} / \mathrm{s})$ silicon chips based OPS/OBS are very promising.

Using a silicon nanowire, we have demonstrated a $160 \mathrm{~Gb} / \mathrm{s}$ packet switch, which can be used in OPS [18]. In this paper, we show that a silicon-based $160 \mathrm{~Gb} / \mathrm{s}$ packet switch can also be used for OBS, and optical buffering could be avoided if a telland-wait (TAW) or TAG protocol is applied. In section II, we describe the working principle of the TAW or TAG protocol and compare the silicon based OBS with the $\mathrm{N} \times \mathrm{N}$ silicon based switch matrix. In section III, the design and the characteristics of the silicon nanowire are presented. In section IV, we describe the working principle of cross phase modulation (XPM) in silicon and its application for packet switching. In addition, we show that the silicon-based $1 \times 1$ all-optical packet switch could be upgraded to $1 \times \mathrm{N}$ all-optical packet switch if a fast tuning laser is used. In Sections V and VI, we show the experimental 


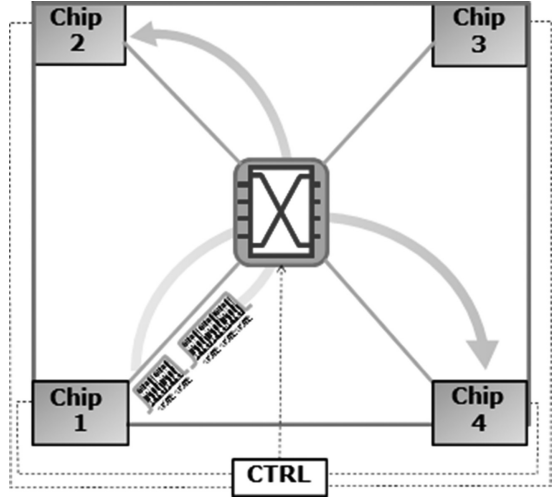

Fig. 1. Short-reach and small-scale network scenario using silicon based OBS.

setup and results of the $160 \mathrm{~Gb} / \mathrm{s}$ all-optical packet switch for OBS. We experimentally demonstrate a $160 \mathrm{~Gb} / \mathrm{s}$ packet switch using an 8.6-mm long silicon nanowire based on XPM. One of four packets at the bit rate of $160 \mathrm{~Gb} / \mathrm{s}$ is switched by an optical control signal. Error free performance (BER $<1 \mathrm{E}-9)$ is achieved for the switched packet.

\section{TAW AND TAG}

In the scenario of short-reach and small-scale interconnects (as shown in Fig. 1), such as interconnections among servers, boards and even chips, optical packets could be stored in the electronic domain using electronic RAM and will not be converted into the optical domain and transmitted until the switching node is ready for the packet. In this case, a two-way reservation protocol such as TAW can be applied [19], [20]. Since the end nodes are close to each other, both BHC and confirmation from the switching node can be sent in the electronic domain with negligible latency and avoiding OEO conversion, which is different in the case of conventional OBS. Another different feature is that the data payload of a burst could be either a short packet or several accumulated long packets, which makes the switching more flexible but requires faster switching speed. In comparison with conventional OPS, which can be used in the large-scale interconnects but requires label processor and packet buffer [21]-[23], our proposed scheme addresses the scenario of short-reach interconnects, and no label is transmitted together with the packet and packet buffer can be avoided.

As shown in Fig. 1, when an ingress end node (EN, e.g., Chip 1) needs to transfer a packet, it first sends a setup message (i.e., BHC) to the control plane. When the control plane receives the setup message, a virtual path will be established towards its egress EN (e.g., Chip 2 or Chip 4) during the data payload transmission if the switching node is free, and then the control plane will send a confirmation message to the ingress EN. Once the ingress EN receives the confirmation message, the data payload stored at the electronic RAM of the ingress EN will be immediately converted into an optical burst and then sent to the egress EN. The virtual path will be automatically released according to the BHC. If more than one ingress EN need to transfer data payload at the same time or the switching node is busy, the data payload at the ingress ENs is still stored

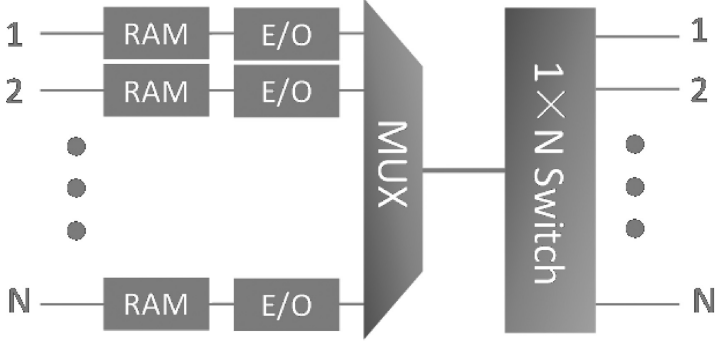

(a)

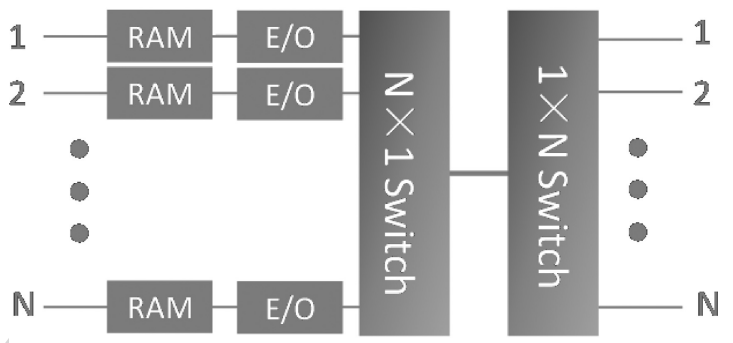

(b)

Fig. 2. Schematic architecture of silicon packet switch based OBS using (a) TAW and (b) TAG.

in the electronic RAM and a waiting list will be established in the control plane. The data payload will wait for the transfer according to the sequence of the list. The sequence of the list depends on the priority based class-of-service. Even if the packet blocking probability rises with higher data load, higher class services experience relatively lower blocking probability compared to lower class services [15]. Fig. 2(a) shows a possible architecture of a silicon packet switch based optical burst switch using TAW protocol. Only one of $\mathrm{N}$ ingress ENs will receive the confirmation message at a time and send data payload to the $1 \times \mathrm{N}$ switch through a multiplexer, which could be a coupler. The $1 \times \mathrm{N}$ switch will switch the data payload to its egress EN according to the BHC.

Another scheme is TAG, which is a one-way reservation protocol and requires no acknowledgement from the switching node before sending the data payload. When an ingress EN has a data payload to transfer, it sequentially sends a setup message to the control plane and an optical burst to the optical switch with a guard time in between. The guard time is at least equal to or more than the time interval needed for setup of a virtual path inside the switching node. This allows the optical switch to be set before the packet arrives. If the switching node is free when it receives the setup message, a virtual path will be set up for the packet transfer and a successful message will be sent back to the ingress EN. If the switching node is busy when it receives the setup message, the optical packet sent from the ingress EN will be discarded and a fail message will be sent back to the ingress EN. If the ingress EN receives the successful message, the electronic RAM storing the data payload will be released. If the ingress EN receives the fail message, it will send the setup message and the optical burst again. Fig. 2(b) shows a possible architecture of silicon packet switch based OBS using TAG protocol. If the control plane receives a setup message from an ingress EN and the switching node is free at the time, the first 

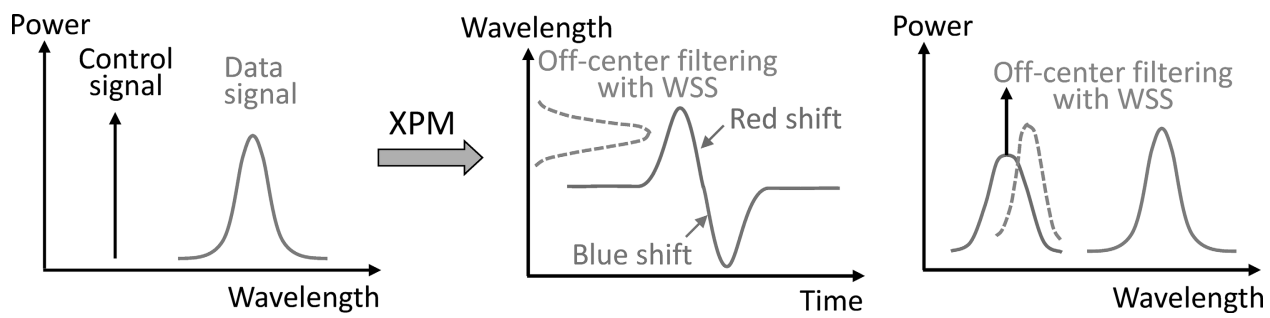

Fig. 3. Operation principle of the XPM in a silicon nanowire with subsequent off-center filtering.

$\mathrm{N} \times 1$ switch will connect to the ingress $\mathrm{EN}$ and allow the data payload to enter, and the second $1 \times \mathrm{N}$ switch will switch to its egress EN according to the BHC. If the switching node is busy when the control plane receives the setup message, the first $1 \times \mathrm{N}$ switch will not connect to the ingress $\mathrm{EN}$ and the data payload sent from the ingress EN will be discarded.

Compared to an $\mathrm{N} \times \mathrm{N}\left(N \in 2^{\mathrm{n}}, n=1,2,3, \ldots\right)$ silicon based switch matrix with a granularity of the optical paths, silicon based OBS with a granularity of optical packets is more flexible and could have smaller footprint and less power consumption. For the $\mathrm{N} \times \mathrm{N}$ switch matrix based on path-independent insertion loss and "switch-and-select" topology, the total number of Mach-Zehnder interferometer (MZI) switches is $2 \times \mathrm{N} \times \mathrm{N}$ and $2 \times \mathrm{N} \times(\mathrm{N}-1)$, respectively [24], [25]. Assuming MZI switches are also used in the $1 \times \mathrm{N}$ switch, as shown in Fig. 2, the total number of MZI switches for the TAW and TAG are $\mathrm{N}-1$ and $2 \times(\mathrm{N}-1)$, respectively. In addition, no intersections are needed for the $1 \times \mathrm{N}$ switch, and therefore there will be no crosstalk.

A main requirement for the silicon packet switch is that the switching speed of the optical switches should be fast enough in order to introduce less latency and lower blocking probability, and therefore the switching time should preferably be no more than a few nanoseconds.

\section{SILICON NANOWIRE}

The key device for the silicon all-optical packet switch is a dispersion engineered 8.6- $\mathrm{mm}$ long silicon straight waveguide, which includes tapering sections for low loss interfacing with optical fiber [26]. The main waveguide section is $\sim 8 \mathrm{~mm}$ long and has a cross-sectional dimension of $240 \mathrm{~nm} \times 450 \mathrm{~nm}$ while the tapering sections are $\sim 0.3 \mathrm{~mm}$ long each. The width at the end of the silicon nanowire is tapered from $450 \mathrm{~nm}$ to a tiny tip end of $40 \mathrm{~nm}$ so that the guided mode will expand into a polymer waveguide, surrounding the silicon-on-insulator (SOI) waveguide and the taper. The device has an SOI structure, with the silicon waveguide placed on a $\mathrm{SiO}_{2} / \mathrm{Si}$ substrate. The measured propagation loss is $4.3 \mathrm{~dB} / \mathrm{cm}$ and the fiber-to-fiber loss of the device is $6.8 \mathrm{~dB}$.

\section{XPM In A Silicon NanOwiRe FOR Packet SWITCH}

XPM is an ultrafast optical Kerr effect, with a response time of a few fs. Fig. 3 shows the operation principle of XPM in a silicon nanowire with subsequent off-center filtering. The pump pulse can modulate the refractive index of the silicon waveguide, which results in phase modulation on the co-propagating con- tinuous wave $(\mathrm{CW})$ probe [27]. The phase modulation will then result in transient chirp on the $\mathrm{CW}$ probe. The leading edges of the pump pulse will generate red-shift chirp, whereas the trailing edge of the pump pulse will generate blue-shift chirp. The blue shifted and red shifted sidebands are generated as a result of the chirp. If an off-center filter is used to extract either the blue shifted sideband or the red shifted sideband, the XPM-induced phase modulation can be converted into amplitude modulation. When an RZ-OOK data signal is used as the pump, the generated sideband can pass through the off-center filter in the presence of a " 1 " bit of the pump, whereas no generated sideband results in no transmission through the off-center filter in the presence of a " 0 " bit of the pump. Using a CW probe, the XPM in silicon with subsequent off-center filtering has been used for forward error correction supported $150 \mathrm{~Gb} / \mathrm{s}$ wavelength conversion, $10 \mathrm{~Gb} / \mathrm{s}$ tuneable wavelength conversion, $40 \mathrm{~Gb} / \mathrm{s}$ regenerative wavelength conversion and $160 \mathrm{~Gb} / \mathrm{s}$ all-optical data modulator [27]-[30]. If the probe is gated in time (with a gating time slightly larger than the packet duration), XPM in silicon with subsequent off-center filtering, can be used for packet switching with ultrafast response time. Fig. 4 shows an illustration of packet switch based on the XPM in silicon with subsequent off-center filtering using gated probe light. For the $1 \times 1$ packet switch, 1 out of 4 data packets $\left(\lambda_{D}\right)$ is switched out at the wavelength of $\lambda_{\mathrm{C}}+\Delta \lambda$ when the control signal $\left(\lambda_{\mathrm{C}}\right)$ is set to be on. When the control signal is off, no light is generated at the wavelength of $\lambda_{\mathrm{C}}+\Delta \lambda$. For the $1 \times \mathrm{N}$ packet switch, a wavelength selective switch (WSS) with different wavelengths at different outputs should be used. When the wavelength of the control signal is fast tuned, the incoming packet could be switched to different outputs of the WSS. The tuning speed of the tunable laser depends on the guard band between packets, which is typically on the order of several nanoseconds to several microseconds. This requirement of tuning speed could be relaxed if the granularity of the packet becomes large, i.e., introducing large enough guard band $(\sim \mu \mathrm{s})$ by switching a long packet or several aggregated short packets. If the first packet of four data packets needs to be switched to port 1 of the WSS, the wavelength of the control signal should be tuned to be $\lambda_{\mathrm{C} 1}$; if the second packet of four data packets needs to be switched to port 4 of the WSS, the wavelength of the control signal should be tuned to be $\lambda_{\mathrm{C} 4}$. Compared to the $1 \times \mathrm{N}$ packet switch based on cascaded MZI switches, which needs $\mathrm{N}-1$ active switch, the XPM based $1 \times \mathrm{N}$ packet switch only needs an active fast tunable laser followed by passive filtering. Based on the $1 \times \mathrm{N}$ packet switch, the TAW and TAG protocol can be realized, as shown in Fig. 2. 


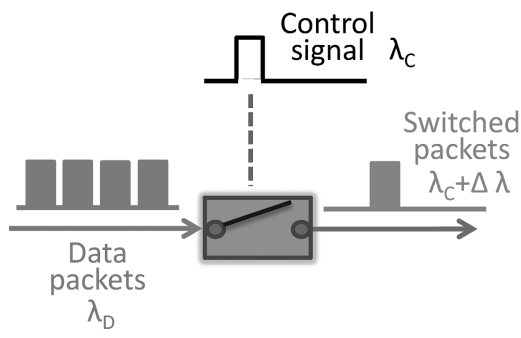

(a)

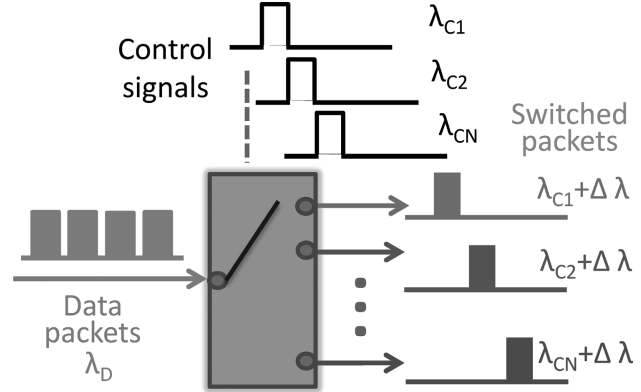

(b)

Fig. 4. Illustration of packet switch. (a) One out of four data packets is switched out using $1 \times 1$ packet switch when the control signal is set to be on; (b) One of the input data packets is switched to different path using $1 \times \mathrm{N}$ packet switch when the wavelength of the control signal is tuned. Blue box: WSS with different wavelengths at different outputs.

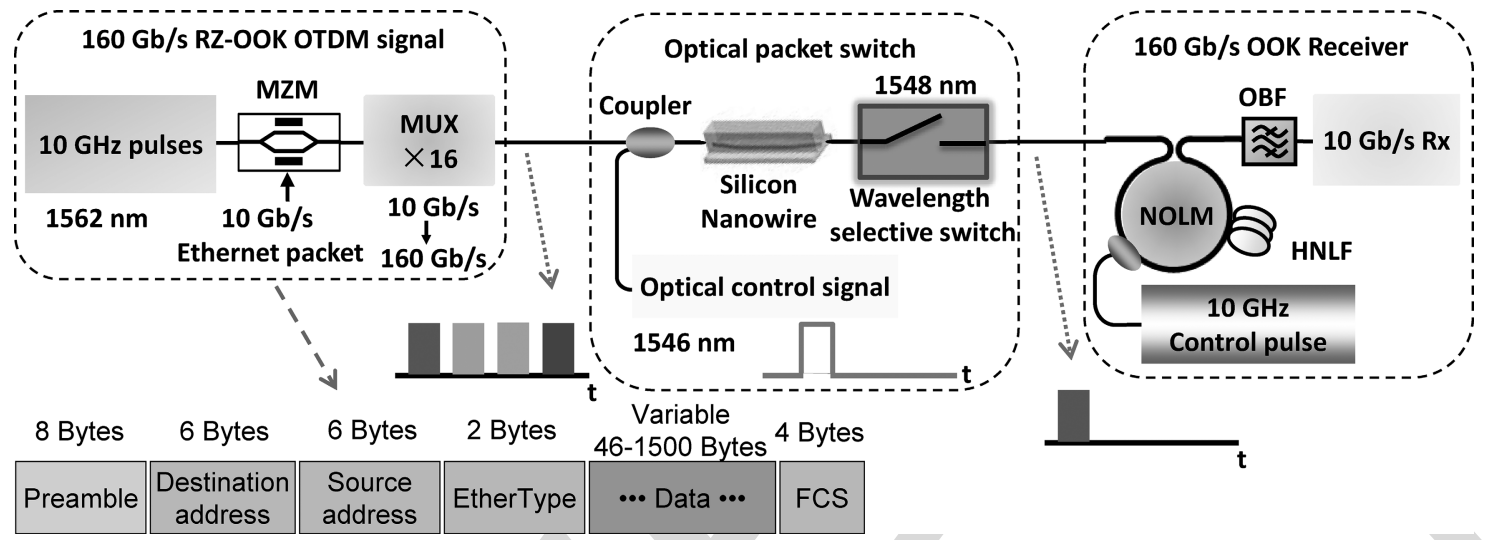

Fig. 5. Experimental setup for the $160 \mathrm{~Gb} / \mathrm{s}$ all-optical packet switch using a silicon nanowire followed by a WSS.

\section{EXPERIMENTAL SETUP}

The experimental setup for the $160 \mathrm{~Gb} / \mathrm{s}$ silicon packet switch is shown in Fig. 5. It mainly includes a $160 \mathrm{~Gb} / \mathrm{s}$ RZ-OOK transmitter, a $1 \times 1$ silicon based packet switch and a $160 \mathrm{~Gb} / \mathrm{s}$ on-off keying (OOK) receiver. The erbium-glass oscillating pulse-generating laser produces $10 \mathrm{GHz}$ pulses at $1542 \mathrm{~nm}$ with a 1.5-ps full-width at half-maximum pulse width. The spectrum of the pulses is broadened in a 400-m dispersion-flattened highly nonlinear fibre (DF-HNLF, dispersion coefficient $\mathrm{D}=$ $-0.45 \mathrm{ps} / \mathrm{nm} / \mathrm{km}$ and dispersion slope $\mathrm{S}=0.006 \mathrm{ps} / \mathrm{nm}^{2} / \mathrm{km}$ at $1550 \mathrm{~nm}$, nonlinear coefficient $\gamma=10.5 \mathrm{~W}^{-1} \mathrm{~km}^{-1}$ ) due to self-phase modulation [31], [32]. The broadened spectrum is filtered at $1562 \mathrm{~nm}$ with a 5-nm optical bandpass filter (OBF) to generate the $10 \mathrm{GHz}$ pulses for the data signal and is also filtered at $1538 \mathrm{~nm}$ using a $1 \mathrm{~nm}$ OBF to obtain the $10 \mathrm{GHz}$ control pulses used in the OTDM demultiplexing. The generated $10 \mathrm{GHz}$ pulses at $1562 \mathrm{~nm}$ are OOK modulated in a MachZehnder modulator (MZM) using a software defined pattern to generate $10 \mathrm{~Gb} / \mathrm{s}$ Ethernet packets. As shown in Fig. 5, the Ethernet packet with a maximum standardized size of 1518 bytes consists of a preamble, a destination address, a source address, an Ethertype, payload data and a frame check sequence [33]. The packets have duration of $2.19 \mu \mathrm{s}$, consisting of $1.22 \mu \mathrm{s}$ of data payload separated by a $0.97 \mu$ s guard band. The generated 10 $\mathrm{Gb} / \mathrm{s}$ Ethernet packet is multiplexed in time using a passive fiberdelay multiplexer $(\mathrm{MUX} \times 16$ ) to generate the $160 \mathrm{~Gb} / \mathrm{s}$ signal.
In the $(1 \times 1)$ silicon based optical packet switch, the gen- 283 erated $160 \mathrm{~Gb} / \mathrm{s}$ optical packet is amplified by an EDFA, then 284 filtered by a $5 \mathrm{~nm} \mathrm{OBF}$ and finally launched into the silicon 285 nanowire through a 3-dB optical coupler. The optical control 286 signal is generated from a CW light at $1546 \mathrm{~nm}$, which is mod- 287 ulated by an electrical control signal in a MZM. The electrical 288 control signal is generated according to the BHC of the burst, 289 which has duration of $1.5 \mu \mathrm{s}$ and repetition rate of $\sim 114 \mathrm{kHz} 290$ in order to switch one of the four packets. The optical control 291 signal is also launched into the silicon nanowire through the 292 second input of the 3-dB coupler. Fig. 6 shows the waveforms 293 of the packets and the control signal. The launched average 294 power for the data and the control are 13.5 and $8.5 \mathrm{dBm}$, respec- 295 tively, which corresponds to the energy consumption of 140 and 296 $44 \mathrm{fJ} / \mathrm{bit}$. Since the total launched power is well below the two 297 photon absorption (TPA) threshold [6], the TPA and resulting 298 carrier effects are negligible. The polarizations of the data signal 299 and control signal are both aligned to transverse-electric polar- 300 izations into the silicon waveguide. The optical packets modu- 301 late the refractive index of the silicon waveguide and generate 302 XPM on the control signal. Only if the control signal presents 303 "1" level and aligned in time with a packet, the control signal will 304 be phase modulated by the optical packet and be converted into 305 an amplitude modulated signal by passing through an off-center 306 filter. Note that the refractive index of the silicon waveguide 307 could also be modulated by an electrical signal [8], therefore, 308 


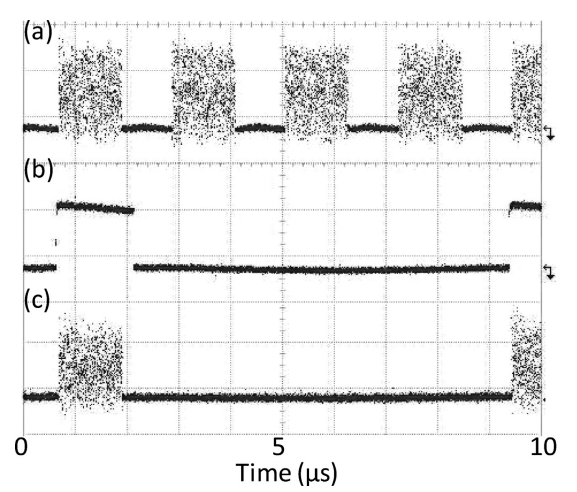

Fig. 6. Oscilloscope traces (a) $160 \mathrm{~Gb} / \mathrm{s}$ optical packets at the input of the silicon nanowire; (b) optical control signal; (c) one out of four packets switched at the output of the silicon nanowire and WSS.

in principle the scheme could also work if the electrical signal is directly employed on the silicon chip. At the output of the silicon nanowire, a WSS with the center wavelength of $1548 \mathrm{~nm}$ is used to filter out the red-shifted sideband of the control signal and select the switched packet.

The 4-to-1 switched $160 \mathrm{~Gb} / \mathrm{s}$ packet was detected using a $160 \mathrm{~Gb} / \mathrm{s}$ OOK receiver, which consists of a nonlinear optical loop mirror (NOLM) based OTDM demultiplexer, a 0.9-nm filter, a photo detector (PD) and an error analyzer. The NOLM is used to OTDM demultiplex the $160 \mathrm{~Gb} / \mathrm{s}$ packet down to $10 \mathrm{~Gb} / \mathrm{s}$ packets based on the XPM in a $50 \mathrm{~m}$ long HNLF. Finally, the demultiplexed $10 \mathrm{~Gb} / \mathrm{s}$ optical packet was detected using a PD and the performance was evaluated using an error analyzer.

\section{EXPERIMENTAL RESULTS}

Fig. 6 shows the dynamic operation of $160 \mathrm{~Gb} / \mathrm{s}$ silicon packet switch. When the control plane receives BHC information, a control signal was generated to drive the silicon based all-optical switch. In this case, one of four packets needs to be switched; therefore, the targeted packet was aligned in time with the optical control signal, as shown in Fig. 6(a) and (b). At the output of the packet switch, the targeted packet was successfully switched with an extinction ratio of $\sim 18 \mathrm{~dB}$, as shown in Fig. 6(c). Fig. 7 shows optical sampling oscilloscope (OSO) eye diagrams of $160 \mathrm{~Gb} / \mathrm{s}$ original packets and 1-of-4 switched packet. The switched packet has an open and clear eye diagram after the packet switch. Actually, some " 0 " level noise on the original packets was also removed after the $1 \times 1$ packet switch, resulting from the regenerative characteristics of the off-center filtering [29].

The spectra at the input and the output of the silicon nanowire are shown in Fig. 8. XPM on the control signal can be clearly seen (modulation peaks around the control signal) at the output of the silicon nanowire. The blue and red shifted sidebands on the optical control signal were generated from the leading edge and trailing edge of the $160 \mathrm{~Gb} / \mathrm{s}$ optical packets with the modulation format of RZ-OOK, respectively. A WSS centered at $1548 \mathrm{~nm}$ is used to filter out the red-shifted probe light to

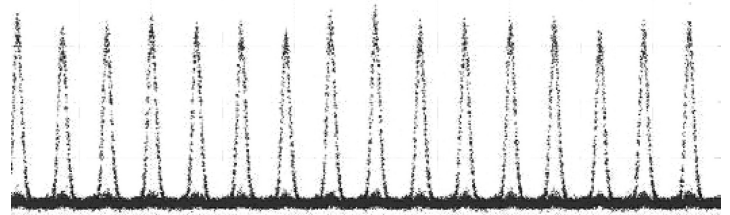

(a)

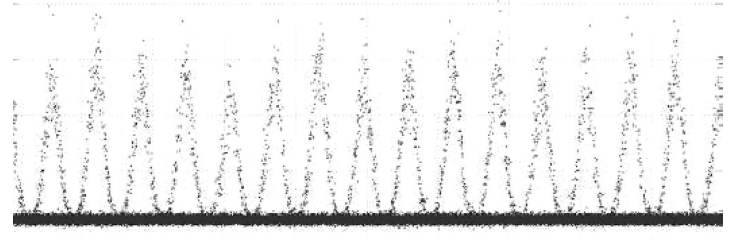

(b)

Fig. 7. (a) OSO eye diagrams of $160 \mathrm{~Gb} / \mathrm{s}$ original packets (b) and 1-of-4 switched packet.

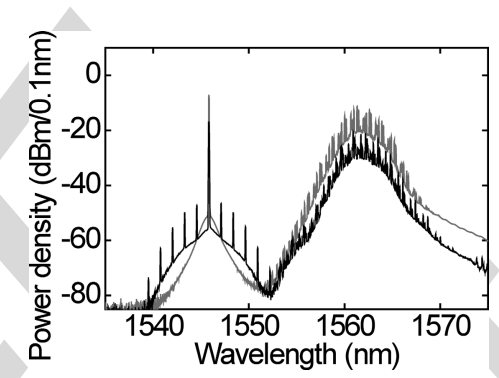

Fig. 8. Optical spectra at the input of the silicon chip (red) and output of the silicon nanowire (black).

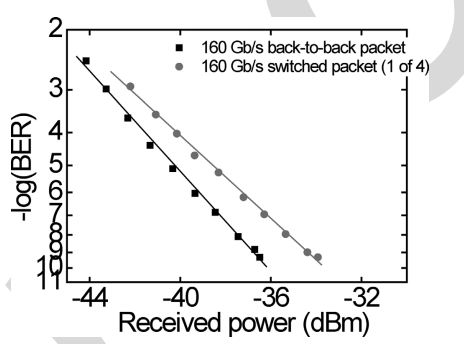

Fig. 9. BER measurements after demultiplexing to $10 \mathrm{~Gb} / \mathrm{s}$ for the $160 \mathrm{~Gb} / \mathrm{s}$ back-to-back packet and for the $160 \mathrm{~Gb} / \mathrm{s}$ 4-to-1 switched packet.

obtain an amplitude modulated $160 \mathrm{~Gb} / \mathrm{s}$ signal and separate the switched packet at $1548 \mathrm{~nm}$ from the original packet at $1562 \mathrm{~nm}$.

The performance of the silicon based $1 \times 1$ all-optical packet switch for the $160 \mathrm{~Gb} / \mathrm{s}$ Ethernet packet was evaluated using BER measurements, as shown in Fig. 9. BER curves are plotted for the $160 \mathrm{~Gb} / \mathrm{s}$ back-to-back packets and for the $160 \mathrm{~Gb} / \mathrm{s}$ 4-to- 1 switched packets. The 4-to- 1 switched $160 \mathrm{~Gb} / \mathrm{s}$ packets achieve an error-free performance (BER $<10^{-9}$ ) with a power penalty of $\sim 2.5 \mathrm{~dB}$ compared to the back-to-back case. The measured penalty is partly attributed to the pulse broadening due to the filtering effect induced by the WSS, and partly attributed to the OSNR degradation after the packet switch due to the limited phase modulation on the control signal. 


\section{DISCUSSION AND CONCLUSION}

We have successfully demonstrated a $160 \mathrm{~Gb} / \mathrm{s}$ silicon based $1 \times 1$ all-optical packet switch, based on XPM in a silicon nanowire. This scheme could be upgraded to a $1 \times \mathrm{N}$ all-optical packet switch if a fast tunable laser is used. The silicon packet switch could be used either for OPS or OBS without optical buffering if the TAW or TAG protocol is used. The 4-to-1 switched $160 \mathrm{~Gb} / \mathrm{s}$ Ethernet packet shows error free performance (BER < 1E-9), and holds great promise for future photonic switching of ultra-fast data signals.

\section{REFERENCES}

[1] Y.A. Vlasov, "Silicon CMOS-integrated nano-photonics for computer and data communications beyond 100G," IEEE Commun. Mag., vol. 50, no. 2, pp. s67-s72, Feb. 2012.

[2] P. W. Coteus, J. U. Knickerbocker, C. H. Lam, and Y. A. Vlasov, "Technologies for exascale systems," IBM J. Res. Develop., vol. 55, no. 5, pp. 14:1-14:12, Sep./Oct. 2011.

[3] IEEE P802.3ba. [Online]. Available: http://www.ieee802.org/3/

[4] IEEE 802.3 industry connections ethernet bandwidth assessment Ad Hoc. [Online]. Available: http://www.ieee802.org/3/ad_hoc/bwa/index.html.

[5] M. A. Foster, A. C. Turner, J. E. Sharping, B. S. Schmidt, M. Lipson, and A. L. Gaeta, "Broad-band optical parametric gain on a silicon photonic chip," Nature, vol. 441, pp. 960-963, 2006.

[6] H. Hu, H. Ji, M. Galili, M. Pu, H. C. H. Mulvad, L. K. Oxenløwe, K. Yvind, J. M. Hvam, and P. Jeppesen, "Ultra-high-speed wavelength conversion in a silicon photonic chip," Opt. Exp., vol. 19, no. 21, pp. 19886-19894, 2011.

[7] C. Batten, A. Joshi, J. Orcutt, A. Khilo, B. Moss, C. W. Holzwarth, M. A. Popovic, H. Li, H. Smith, J. L. Hoyt, F. X. Kartner, R. J. Ram, V. Stojanovic, and K. Asanovic, "Building many-core processor-toDRAM networks with monolithic CMOS silicon photonics," IEEE Micro, vol. 29, no. 4, pp. 8-21, Jul./Aug. 2009.

[8] P. Dong, X. Liu, C. Sethumadhavan, L. L. Buhl, R. Aroca, Y. Baeyens, and Y. Chen, "224-Gb/s PDM-16-QAM modulator and receiver based on silicon photonic integrated circuits," presented at the Opt. Fiber Commun. Conf., Nat. Fiber Opt. Eng., Anaheim, CA, USA, 2013, Paper PDP5C.6.

[9] H. Ji, M. Pu, H. Hu, M. Galili, L. K. Oxenløwe, K. Yvind, J. M. Hvam, and P. Jeppsen, "Optical waveform sampling and error-free demultiplexing of $1.28 \mathrm{~Tb} / \mathrm{s}$ serial data in a nanoengineered silicon waveguide," J. Lightw. Technol., vol. 29, no. 4, pp. 426-431, Feb. 2011.

[10] H. C. Hansen Mulvad, E. Palushani, H. Hu, H. Ji, M. Lillieholm, M. Galili, A. T. Clausen, M. Pu, K. Yvind, J. M. Hvam, P. Jeppesen, and L. K. Oxenløwe, "Ultra-high-speed optical serial-to-parallel data conversion by time-domain optical Fourier transformation in a silicon nanowire," Opt. Exp., vol. 19, pp. B825-B835, 2011.

[11] J. Leuthold, C. Koos, and W. Freude, "Nonlinear silicon photonics," Nature Photon., vol. 4, pp. 535-544, 2010.

[12] W. Stallings, High-Speed Networks. Englewood Cliffs, NJ, USA: PrenticeHall, 1998.

[13] S. Yao, S. J. B. Yoo, B. Mukherjee, and S. Dixit, "All-optical packet switching for metropolitan area networks: Opportunities and challenges," IEEE Commun. Mag., vol. 39, no. 3, pp. 142-148, Mar. 2001.

[14] C. M. Qiao and M. S. Yoo, "Optical burst switching (OBS)—A new paradigm for an optical Internet," J. High Speed Netw., vol. 8, no. 1, pp. 69-84, 1999.

[15] M. Yoo, C. M. Qiao, and S. Dixit, "Optical burst switching for service differentiation in the next-generation optical internet," IEEE Commun. Mag., vol. 39, no. 2, pp. 98-104, Feb. 2001.

[16] S. J. B. Yoo, "Optical packet and burst switching technologies for the future photonic internet," J. Lightw. Technol., vol. 24, no. 12, pp. 4468 -4492 , Dec. 2006.

[17] F. Gomez-Agis, H. Hu, J. Luo, H. C. H. Mulvad, M. Galili, N. Calabretta, L. K. Oxenløwe, H. J. S. Dorren, and P. Jeppesen, "Optical switching and detection of $640 \mathrm{Gbits} / \mathrm{s}$ optical time-division multiplexed data packets transmitted over $50 \mathrm{~km}$ of fiber," Opt. Lett., vol. 36, pp. 3473-3475, 2011.
[18] H. Hu, H. Ji, M. Galili, M. Pu, K. Yvind, P. Jeppesen, and L. K. Oxenløwe, "160 Gbit/s optical packet switching using a silicon chip," in Proc. IEEE Photon. Conf., Sep. 2012, pp. 915-916.

[19] P. E. Boyer and D. P. Tranchier, "A reservation principle with applications to the ATM traffic control," Comput. Netw. ISDN Syst., vol. 24, pp. 321-334, 1992.

[20] A. Detti and M. Listanti, "Application of tell \& Go and tell \& wait reservation strategies in a optical burst switching network: A performance comparison," in Proc. IEEE Int. Conf. Telecommun., 2001, vol. 2, pp. $540-548$.

[21] N. Calabretta, R. P. Centelles, S. Di Lucente, and H. J. S. Dorren, "On the performance of a large-scale optical packet switch under realistic data center traffic," J. Opt. Commun. Netw., vol. 5, no. 6, pp. 565-573, Jun. 2013.

[22] S. Di Lucente, N. Calabretta, J. A. C. Resing, and H. J. S. Dorren, "Scaling low-latency optical packet switches to a thousand ports," J. Opt. Commun. Netw., vol. 4, no. 9, pp. A17-A28, Sep. 2012.

[23] H. J. S. Dorren, S. Di Lucente, J. Luo, O. Raz, and N. Calabretta, "Scaling photonic packet switches to a large number of ports [invited]," J. Opt. Commun. Netw., vol. 4, no. 9, pp. A82-A89, Sep. 2012.

[24] L. Chen and Y. K. Chen, "Compact, low-loss and low-power $8 \times 8$ broadband silicon optical switch," Opt. Exp., vol. 20, no. 17, pp. 18977-18985, 2012.

[25] K. Suzuki, K. Tanizawa, T. Matsukawa, G. Cong, S. H. Kim, S. Suda, M. Ohno, T. Chiba, H. Tadokoro, M. Yanagihara, Y. Igarashi, M. Masahara, and H. Kawashima, "Ultra-compact Si-wire $8 \times 8$ strictly-non-blocking PILOSS switch," presented at the Eur. Conf. Exhib. Opt. Commun., London, U.K., 2013, Paper PD2.D.2.

[26] M. Pu, L. Liu, H. Ou, K. Yvind, and J. M. Hvam, "Ultra-low-loss inverted taper coupler for silicon-on-insulator ridge waveguide," Opt. Cотmиn., vol. 283, pp. 3678-3682, Oct. 2010.

[27] H. Hu, J. Dahl Andersen, A. Rasmussen, B. M. Sørensen, K. Dalgaard, M. Galili, M. Pu, K. Yvind, K. J. Larsen, S. Forchhammer, and L. K. Oxenløwe, "Forward error correction supported $150 \mathrm{Gbit} / \mathrm{s}$ error-free wavelength conversion based on cross phase modulation in silicon," Opt. Exp., vol. 21, pp. 3152-3160, 2013.

[28] W. Astar, J. B. Driscoll, X. Liu, J. I. Dadap, W. M. J. Green, Y. Vlasov, G. M. Carter, and R. M. Osgood, "Tunable wavelength conversion by XPM in a silicon nanowire, and the potential for XPM-multicasting," $J$. Lightw. Technol., vol. 28, no. 17, pp. 2499-2511, Sep. 2010.

[29] A. S. Jensen, H. Hu, H. Ji, M. Pu, L. H. Frandsen, and L. K. Oxenløwe, "All-optical $40 \mathrm{Gbit} / \mathrm{s}$ regenerative wavelength conversion based on crossphase modulation in a silicon nanowire," presented at the IEEE 18th OptoElectron. Commun. Conf. Held Jointly Int. Photon. Switching, Kyoto, Japan, 2013, Paper ThM1-2.

[30] H. Hu, M. Pu, H. Ji, M. Galili, H. C. H. Mulvad, K. Yvind, J. M. Hvam, P. Jeppesen, and L. Oxenlowe, "160 Gb/s silicon all-optical data modulator based on cross phase modulation," presented at the Optical Fiber Commun. Conf. Expo. Nat. Fiber Opt. Eng. Conf., Los Angeles, CA, USA, 2012, Paper OM2E.2.

[31] H. Hu, H. C. H. Mulvad, M. Galili, E. Palushani, J. Xu, A. T. Clausen, L. K. Oxenløwe, and P. Jeppesen, "Polarization-insensitive $640 \mathrm{~Gb} / \mathrm{s} \mathrm{de}$ multiplexing based on four wave mixing in a polarization-maintaining fibre loop," J. Lightw. Technol., vol. 28, no. 12, pp. 1789-1795, Jun. 2010.

[32] H. Hu, E. Palushani, M. Galili, H. C. H. Mulvad, A. Clausen, L. K. Oxenløwe, and P. Jeppesen, "640 Gbit/s and $1.28 \mathrm{Tbit} / \mathrm{s}$ polarisation insensitive all optical wavelength conversion," Opt. Exp., vol. 18, pp. 99619966, 2010.

[33] H. Hu, J. L. Areal, H. C. H. Mulvad, M. Galili, K. Dalgaard, E. Palushani, A. Clausen, M. S. Berger, P. Jeppesen, and L. K. Oxenløwe, "Synchronization, retiming and time-division multiplexing of an asynchronous 10 Gigabit NRZ Ethernet packet to terabit Ethernet," Opt. Exp., vol. 19, pp. B931-B937, 2011. 
